RICYDE. Revista Internacional de Ciencias del Deporte doi: $10.5232 /$ ricyde

Rev. int. cienc. deporte

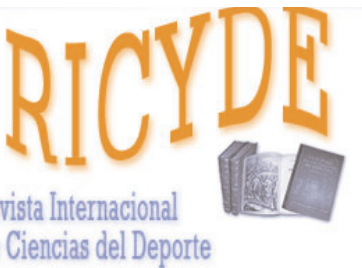

RICYDE. Revista Internacional de Ciencias del Deporte VOLUMEN XI - AÑO XI

Páginas:186-191 ISSN:1885-3137 No 40 - Abril - 2015

\title{
David Falk: Algunas cuestiones abiertas para el marketing deportivo David Falk: Some open questions for the sports marketing arena
}

\author{
José A. Martínez
}

Universidad Politécnica de Cartagena, España

\begin{abstract}
Resumen
David Falk, el que fuera el representante del jugador de baloncesto Michael Jordan, es una figura de referencia para el marketing deportivo. Falk contribuyó decisivamente a revitalizar el marketing deportivo, ayudó a construir lo que probablemente sea la relación comercial más importante del deporte (Jordan-Nike), y llevó hasta el extremo la filosofía de que en la negociación lo importante es que tu cliente llegue a conseguir el máximo dinero posible, más allá de que ese sea su valor de mercado o que el propio mercado pueda admitir esa inflación. En este artículo se repasan algunos de los hechos más destacados de su vida profesional y, desde una perspectiva crítica, se relacionan con varias cuestiones abiertas que el mundo académico y profesional del deporte continúa discutiendo.
\end{abstract}

Palabras clave: David Falk; negociación; deporte profesional; marketing deportivo.

\begin{abstract}
David Falk, former agent of the basketball player Michael Jordan, is a figure of reference for sports marketing. Falk was instrumental in revitalizing the sports marketing. He helped to build what is probably the most important relationship in sport business (Jordan-Nike), and strongly defended a radical philosophy of negotiation, where the most important is the maximization of client gains, regardless their true market value or if the market can admit such inflation. This article reviews some of the highlights of his career facts and, from a critical perspective, discusses several open issues related to the academic and professional world of sports. To achieve this aim, several recent contributions to the marketing and sports literature are commented.
\end{abstract}

Key words: David Falk; negotiation; professional sport; sport marketing.

Correspondencia/correspondence: José A. Martínez García

Facultad de Ciencias de la Empresa. Universidad Politécnica de Cartagena. España

Email: josean.martinez@upct.es 


\section{Introducción}

"El agente de Michael Jordan". David Falk es identificado y será siempre recordado en el mundo del deporte por esas palabras que suelen apostillar su nombre. Es su tagline, la mejor manera de definir su marca personal. Falk fue el hombre que ayudó decisivamente a que Jordan y Nike revolucionaran el marketing deportivo, allá por 1984.

Esa historia ha sido narrada muchas veces por otros (ej. Katz, 1994; Smit, 2008, Strasser \& Bercklund, 1991), y también por el propio Falk (Falk, 2009). La firma de ese contrato fue fruto del trabajo que Falk y la agencia para la que trabajaba, ProServ, estaban realizando desde hacía una década. Entre ellas destacan las buenas relaciones con la Universidad de North Carolina a través de su entrenador Dean Smith, o la ligazón a Nike gracias a la firma de, por ejemplo, Adrian Dantley, en 1976. Aunque Jordan prefería Adidas, y había jugado en su etapa universitaria con zapatillas Adidas y Converse, las ofertas de esas compañías no se ajustaban a lo que Falk tenía en mente, y eso que estaba en consonancia con lo que muchos de los grandes jugadores cobraban entonces, y además, había que tener en cuenta que Jordan ni siquiera fue número 1 del draft, sino número 3.

Pero Falk quería otra cosa, y Nike necesitaba, asimismo, reactivar su marca. La enseña de Oregón había crecido muchísimo a finales de la década de los 70 y comienzos de los 80 , hasta convertirse en la marca más cool para la juventud norteamericana, icono de rebeldía y cambio. Sin embargo, Reebok le estaba comiendo terreno desde 1982, enfocándose principalmente en las mujeres y el aeróbic, y además Nike estaba teniendo problemas financieros (había crecido demasiado rápido y la estructura de la empresa no se pudo ajustar adecuadamente). Para colmo, a comienzos de esa década tenía una presencia en la $N B A$ que, aunque era importante, no era tan preponderante como la de Converse y Adidas. De este modo, Falk y Nike decidieron arriesgar al máximo, y posicionar al jugador como el referente del baloncesto mundial para los próximos años. Esto, desde luego, parecía a priori una temeridad en una $N B A$ dominada también por jóvenes estrellas como Magic Johnson y Larry Bird. Pero Falk consiguió para su representado un contrato de 2.5 millones de dólares por 5 años, una línea de zapatillas "Air Jordan" y los correspondientes royalties. Falk aprovechó la experiencia adquirida anteriormente en la negociación que realizó para uno de sus clientes, James Worthy, con New Balance en 1982, donde consiguió un contrato de 1.2 millones de dólares por 8 años, algo inaudito hasta ese momento. Pero las cifras del contrato de Jordan eran de otro mundo.

Desde entonces el poder de Falk creció exponencialmente en la $N B A$, y su nombre se ligó inexorablemente al éxito que Jordan consiguió como jugador y como embajador de la marca Nike. Falk empezó a tener una nómina de clientes renombrados (Patrick Ewing, Alonzo Mourning, Reggie Miller, Allen Iverson...), y estuvo detrás de las diferentes negociaciones que los jugadores y la $N B A$ realizaron durante los cierres patronales de 1995 y 1998. Si Falk, Jordan y Nike, y ese mágico contrato de 1984 representan un tótem en el inconsciente colectivo del marketing deportivo, sobre la figura del agente se ciernen diversas cuestiones que permanecen todavía sujetas a discusión.

\section{El jugador como embajador de múltiples marcas}

Se le atribuye a Falk la conversión de Jordan en una especie de primer "hombre-anuncio" del baloncesto al apadrinar, además de a Nike, marcas como Coca-Cola, Gatorade, Chevrolet, Wheaties o McDonald's, entre otras. Pero Jordan no fue el pionero. Años atrás, el jugador de los Globetrotters, Meadowlark Lemon, quien también tenía un contrato con Nike, había hecho anuncios para Footlocker, Burger King, Pepsi Cola, Dr. Pepper, Band-Aid, y muchos más (Martínez, 2014a). 
La cuestión relevante para el marketing deportivo reside en analizar si esos múltiples apadrinamientos son realmente efectivos. Cuando existe congruencia entre el jugador y la marca anunciada la efectividad se incrementa (Braunstein-Minkove, Zhang y Trail, 2011; Rossiter y Smidts, 2012), pero cuando la relación jugador-marca no es congruente la efectividad disminuye al desvirtuarse la credibilidad del deportista (Chien, Cornwell y Pappu, 2011; Tripp, Jensen y Carlson, 1994).

El caso de Jordan fue un caso especial, como sugieren Ding, Molchanov y Stork (2011), por su dimensión como deportista. Por ejemplo, cuando Jordan anunció en 1995 que volvía a las canchas de juego tras su primera retirada, no sólo se incrementaron las ventas de Nike, sino también las del resto de marcas apadrinadas por el jugador (Lear, Runyan y Whitaker, 2009).

Otros jugadores, como Shaquille O’Neal, siguieron esa misma senda de múltiples contratos publicitarios. En España, más recientemente, Pau Gasol, ha anunciado a Banco Popular, Costa Blanca, San Miguel, Play Station, Time Force...Entonces, ¿por qué muchas marcas no vinculadas al deporte siguen invirtiendo ingentes cantidades de dinero en la contratación de un deportista cuando éste ya está realizando o ha realizado varias campañas de publicidad para otras marcas? ¿Sigue siendo una estrategia efectiva aunque la literatura de marketing sugiera lo contrario? Es obvio que se necesita investigar más sobre esta cuestión. Y es que probablemente no se debería tomar el caso de Jordan como referente debido a su excepcionalidad como deportista.

\section{El negocio por encima de todo}

Las maniobras de Falk influyeron sin duda en que los contratos de los jugadores empezaran a inflarse. El comisionado David Stern, Magic, Bird y el propio Jordan convirtieron a la NBA en una empresa transnacional de entretenimiento, por lo que el crecimiento de los contratos millonarios fue acompañado también de una expansión y agigantamiento del negocio por parte de la Liga. Sin embargo, ¿dónde quedan los valores más nobles del deporte en este contexto? Y es que, por ejemplo, el negocio se defiende incluso desde los ámbitos teóricamente más objetivos, como el estamento arbitral: Price, Remer y Stone (2012) muestran que, como los partidos más igualados son más atractivos para el público (y por ende para el negocio), los árbitros sistemáticamente actúan para mantener esos partidos lo más ajustados posible, sobre todo si el partido es televisado para todo el país (Wang y col., 2014).

Esa visión neo-capitalista, en ocasiones llevada al extremo, y que es la que el propio Falk admite como el fundamento de su propia filosofía, deja de nuevo muchos interrogantes para el marketing deportivo. Por ejemplo, algunos de los jugadores más grandes de todos los tiempos que formaban el Dream Team en los JJOO de Barcelona'92 se comportaron como auténticos mercenarios al negarse a recoger la medalla de oro vestidos con un chándal Reebok, marca con la que el equipo de Estados Unidos tenía un acuerdo, y rival directo de Nike y Converse. Michael Jordan, Charles Barkley y Magic Johnson, encabezaron un motín que llevaron gestando desde antes que comenzaran incluso los Juegos (Katz, 1994). De ese modo, una de las principales preocupaciones que esos jugadores tenían en mente durante toda la competición, era no vestir durante sólo unos minutos un chándal de la marca rival de las que les pagaba su contrato de ropa y zapatillas. Como indica Martínez (2014b), las paradojas que se dan asociadas a este tipo de casos son numerosas; por ejemplo, en la $N B A$ todos los jugadores se visten de Adidas en su ropa de calentamiento, ya que la marca alemana tiene un contrato con la $N B A$ desde hace varios años. Por tanto, jugadores icónicos para marcas como Nike, como Kobe Bryant y LeBron James, aparecen continuamente ataviados con material de Adidas. En 1992, esos mismos jugadores amotinados vestían disciplinadamente en cada partido en la $N B A$ ropa de calentamiento Champion, que era la marca que vestía a los equipos de la NBA entonces, y que también era rival de Nike, Converse y demás enseñas. 
Falk fue el que tuvo la idea de que finalmente salieran a recoger la medalla tapando el logo de Reebok con banderas de Estados Unidos. Y como sólo había tres banderas disponibles, el resto de jugadores se abrió la chaqueta de chándal para tapar el símbolo de la marca. Pero probablemente esa efervescencia desmedida de los contratos de los jugadores con las marcas deportivas en la segunda mitad de los 80 y comienzos de los 90, de la que Falk era en parte responsable, hizo vivir ese esperpéntico momento; uno de los episodios más lamentables asociados a la tensión entre valores deportivos y negocio. La película Jerry Maguire, estrenada en 1996, y protagonizada por Tom Cruise, ejemplifica a la perfección ese universo.

Ese motín finalmente favoreció a Reebok, que se vio premiado con una publicity extraordinaria, pero, ¿realmente ese tipo de comportamientos perjudica a la imagen de los jugadores y la de las marcas que los patrocinan? A priori podría pensarse que es así. El propio Falk admitió que cuestionó a Michael Jordan su relación con Al Wood (Falk, 2009), un jugador inmerso en diversos problemas con las drogas. Pero hay muchos ejemplos en la realidad que muestran que ello no es tan obvio (ver Martínez, 2014a), que conductas que podrían etiquetarse como "poco deportivas" no alejan algunas marcas de algunos deportistas y que existe un doble rasero que las propias marcas aplican para castigar este tipo de comportamientos.

¿Los deportistas tienen que ser un modelo de comportamiento ejemplar? La respuesta a esta pregunta desde el punto de vista de marketing es rotunda: No. Las marcas saben que los "chicos malos" venden, pero la cuestión es hasta qué punto la obsesión por conseguir el mejor contrato posible penaliza la percepción que el público tiene de esos deportistas. Charles Barkley a comienzos de los 90 dejó muy claro que el no tenía que ser un ejemplo para la gente, y que tenía 2 millones de razones para no llevar Reebok (en referencia a la cuantía de su contrato con Nike). Si, según Falk (2009), Michael Jordan habría jugado gratis al baloncesto, ¿por qué Jordan se molestó con el dueño de los Bulls, Jerry Reindsorf, por negarse a subir su contrato de ¡30 millones de dólares! en 1996 a 36 en 1997? Entonces, ¿afecta en alguna medida a las marcas que apadrinan a estos deportistas la percepción que el público tiene de éstos sobre su sed desmedida de dinero? O, como sugiere Llopis-Goig (2014), ¿el público es capaz de distanciarse de todos los aspectos que no son estrictamente deportivos, tomando el consumo de un espectáculo deportivo como si fuera una película en el cine?

Falk se comportó como Jerry Maguire, antes de que Tom Cruise escribiera esa declaración de intenciones que lo llevó a perder todo su portafolio de clientes, excepto a uno. La emocionante transformación de Cruise en esa película no escondió la cruda realidad del mensaje de que el "negocio" está por encima de todo: “Enséñame la pasta!”.

Saltando del cine nuevamente al mundo real, Sony Vaccaro, quien recomendó a Nike el fichaje de Jordan, y quien fue creador del Campus ABCD, comentaba al respecto (Lypsite, 1997): "Lo que yo hago es moralmente reprobable, es muy malo para el baloncesto, para los chicos, para el país. Nike es la gran nube negra que lo cubre todo, envenena la mente de los chicos y arruina el juego. Ahora estamos pagando a entrenadores de instituto para que podamos atar a sus chicos, por lo que capturamos las mentes y las almas de la gente. Esas son las reglas, pero debemos cambiarlas".

\section{Falk tras la retirada de Jordan}

Falk dejó ProServ en 1992, aunque no se fue por principios éticos como Cruise en Jerry Maguire, sino porque se consideraba mal pagado, y también a diferencia de la película sí que se llevó a los clientes consigo. Fundó su propia agencia, FAME, con la que siguió alimentando su fama de voraz negociador. En 1998 Falk vendió su agencia al grupo 
empresarial $S F X$, que a su vez se encargó de adquirir más agencias en los años subsiguientes. Falk seguía moviendo los hilos allí, con cientos de clientes, hasta que un nuevo proceso de adquisiciones corporativas le llevó a refundar de nuevo FAME en 2007.

En la actualidad, Falk cuenta con un corto y discreto portafolio de jugadores, y aunque es cierto que algunas de sus declaraciones resuenan todavía fuertemente en el universo mediático de la $N B A$, su influencia ha decaído tras la retirada definitiva de Michael Jordan en 2001.

En 2008, Falk donó 5 millones de dólares para crear el David B. Falk Center for Sport Management y más tarde 15 millones para crear el David B. Falk College of Sport and Human Dynamics, ambos en la Universidad de Siracusa, donde se graduó a comienzos de los 70. Desde el punto de vista de marketing se podría identificar esta acción como un patrocinio de naming right. Falk tiene entonces una Facultad con su nombre, y esa labor "filantrópica" (por otro lado loable) tiene unos réditos a nivel de imagen que son innegables.

Este tipo de acuerdos de patrocinio (algunos lo ven como mecenazgo) en las universidades no es algo que haya inventado Falk, ya que es bastante habitual en Estados Unidos. Pero sí que deja claramente fijado los principios más básicos del marketing para las empresas: dar algo siempre a cambio de algo. Y es que, como Cherrier y Murray (2004) comentan, el marketing no tiene nada de virtuoso; esta forma de marketing que Falk practica para sí mismo ciertamente no lo es. Crea valor para los demás, sí, pero también valor comercial para sí mismo. Es un negocio, una actividad de relaciones públicas, un patrocinio, pero no es filantropía.

\section{Conclusión}

Podemos afirmar que Falk contribuyó decisivamente a revitalizar el marketing deportivo, ayudó a construir lo que probablemente sea la relación comercial más importante del deporte (Jordan-Nike), y llevó hasta el extremo la filosofía de que en la negociación lo importante es que tu cliente llegue a conseguir el máximo dinero posible, más allá de que ese sea su valor de mercado o que el propio mercado pueda admitir esa inflación. Su inteligencia, sus éxitos y su visión de negocio son poco cuestionables. Cabe la discusión de si otra forma distinta de acometer ese trabajo sería posible. Como Tom Cruise decía a su mujer, Renée Zellweguer, al final de Jerry Maguire, para tratar de restablecer su relación: "Hola, estoy buscando a mi esposa (...) Vivimos en un mundo cínico. Un mundo cínico. Y trabajamos en un negocio de muchos competidores...", tal vez el paradigma de trabajo de Falk sea la única fórmula posible. O tal vez otra forma de hacer las cosas sea también viable e igualmente valiosa para todas las partes implicadas. Al fin y al cabo, Zellweguer cortaba a Cruise en su discurso, y le decía simplemente: "Cállate. Cállate. Ya me tenías con el hola".

\section{Referencias}

Braunstein-Minkove, J. R.; Zhang, J. J., \& Trail, G. T. (2011). Athlete endorser effectiveness: Model development and analysis. Sport, Business, and Management: An International Journal, 1, 93-114. http://dx.doi.org/10.1108/20426781111107199

Cherrier, H. \& Murray, J. B. (2004). The sociology of consumption, the hidden facet of marketing. Journal of Marketing Management, 20, 509-525. http://dx.doi.org/10.1362/0267257041323954

Chien, P. M.; Cornwell, T.B., \& Pappu, R. (2011). Sponsorship portfolio as a brandimage creation strategy. Journal of Business Research, 64, 142-149. http://dx.doi.org/10.1016/j.jbusres.2010.02.010 
Ding, H., A.; Molchanov, E., \& Stork P. A. (2011). The value of celebrity endorsements: A stock market perspective. Marketing Letters, 22(2), 147-163. http://dx.doi.org/10.1007/s11002-010-9117-y

Falk, D. (2009) The bald truth. New York: Pocket books.

Katz, D. (1994). Just Do It: The Nike Spirit in the Corporate World. Hollbrook, MA: Adams Media Corporation.

Lear, K. E.; Runyan, R. C., \& Whitaker, W. H. (2009). Sports celebrity endorsements in retail products advertising. International Journal of Retail \& Distribution Management, 37(4), 308-321. http://dx.doi.org/10.1108/09590550910948547

Llopis-Goig, R. (2014). Football clubs' ownership and management. The fans' perspective. RICYDE. Revista Internacional de Ciencias del Deporte, 35(10), 16-33. http://dx.doi.org/10.5232/ricyde2014.03502

Lypsite, R. (1997, julio 6). If it's gotta be the shoes, he's gotta be the guy. The New York Times. Recuperado el 19 de diciembre de 2014 disponible en: http://www.nytimes.com/1997/07/06/sports/if-it-s-gotta-be-the-shoes-he-s-gottabe-the-guy.html

Martínez, J. A. (2014). Publicidad, baloncesto y zapatillas. Almería: Editorial Círculo Rojo.

Martínez, J. A. (2014). The paradoxical marketing of sports equipment brands. RICYDE. Revista Internacional de Ciencias del Deporte, 35(10), 1-3. http://dx.doi.org/10.5232/ricyde2014.035

Price, J.; Remer, M., \& Stone, D. F. (2012). Subperfect game: Profitable biases of NBA referees. Journal of Economics \& Management Strategy, 21, 271-300. http://dx.doi.org/10.1111/j.1530-9134.2011.00325.x

Rossiter, J. R. \& Smidts, A. (2012). Print advertising: Celebrity presenters. Journal of Business Research, 65, 874-879. http://dx.doi.org/10.1016/j.jbusres.2011.01.010

Smit, B. (2008). Sneaker wars. New York: Harper Collins.

Strasser, J. B. \& Becklund, L. (1991). The story of Nike and the men who played there. San Diego: Harcourt Brace Jovanovich.

Tripp, C.; Jensen, T. D., \& Carlson, L. (1994). The effects of multiple product endorsements by celebrities on consumers' attitudes and intentions. Journal of Consumer Research, 20(4), 535-547. http://dx.doi.org/10.1086/209368

Wang, Y.; Hilsman, W.; Caudill, S. B., \& Mixon, F. G., Jr. (2014). Television coverage and outcome uncertainty in sports: Empirical evidence from the NBA and WNBA. RICYDE. Revista Internacional de Ciencias del Deporte, 35(10), 34-45. http://dx.doi.org/10.5232/ricyde2014.03503 